\title{
PRIMERAS MUJERES PERIODISTAS DE SAN SEBASTIÁN A CÁDIZ. LOS EJEMPLOS DE CARMEN DE BURGOS Y JOSEFINA CARABIAS, PIONERAS EN EL PERIODISMO Y EN LA RADIO, RESPECTIVAMENTE ${ }^{1}$
}

\author{
Antonio Torres Flores ${ }^{2}$
}

Primeras mujeres periodistas de San Sebastián a Cádiz. los ejemplos de Carmen de Burgos y Josefina Carabias, pioneras en el periodismo y en la radio, respectivamente

Resumen: El recorrido por la Igualdad sigue siendo difícil y merece la pena plantearse las dificultades de aquellas pioneras con las de la sociedad de 2016. El concepto de inferioridad femenina, física e intelectual, fue una creencia generalizada, apoyada por un discurso religioso. Una de las precursoras del feminismo, Carmen de Burgos Seguí "Colombine" (1867-1932), se convierte en la primera mujer que sededicó al ejercicio profesional del periodismo en España. En la radio esa figura de pionera la representa Josefina Carabias (1908-1980). Carabias entró a trabajar en La Palabra como redactora de ese primer informativo de la historia de la radio en España en 1932. Carabias es la primera mujer española que tuvo como tarea principal el periosdismo, a diferencia de sus antecesoras, que eran escritoras o colaboradoras. Carabias tenía una relación laboral de plantilla en Unión Radio Madrid, génesis de lo que es en la actualidad la Cadena SER. Su trabajo fue incansable hasta el momento de su desaparación. En los años cincuenta se convitió en una de las primeas corresponsales en el extranjero.

Palabras clave: Carmen de Burgos, Josefina Carabias, periodismo, radio.

First Women Journalists from San Sebastian and Cadiz: Journalism and Radio Forerunners Carmen de Burgos and Josefina Carabias

Abstract: The path towards equality is still a difficult one and this is why it is important to consider the struggles of pioneer women against the backdrop of our current society. The idea of female physical and intellectual inferiority was a general belief which found support in religious discourse. One of the feminist forerunners, Carmen de Burgos Seguí "Colombine" (1867-1932) became the first Spanish women who devoted her career to journalism and Josefina Carabias (1908-1980) was the first radio journalist in Spain. Carabias started her career as the editor of La Palabra, the first radio news programme in Spain in 1932. She, thus, became the first woman to have a central role as a journalist since her precursors worked mainly as writers or collaborators. Carabias held a fixed position in Unión Radio Madrid, currently known as Cadena Ser. She works tirelessly until the end. In the 1950s she became one of the first women correspondents abroad.

Keywords: Carmen de Burgos, Josefina Carabias, journalism, radio.

\footnotetext{
${ }^{1}$ Fecha de receción: 24/11/2016.

Fecha de aceptación: 22/12/2016.

${ }^{2}$ Director Territorial de Canal Sur Radio y Televisión (RTVA) en Almería. Miembro del Grupo de Investigación Estudios del Tiempo Presente (ETP) y del Centro de Investigación Comunicación y Sociedad (CySOC), ambos vinculados a la Universidad de Almería; $₫$ antoniotorres@yahoo.es
} 


\section{Introducción}

El siglo XIX fue el de las revoluciones sociales, de la expansión de las ideas igualitarias surgidas en la Revolución Francesa y también hubo mujeres españolas que participaron en las nuevas ideas que se iban imponiendo, apoyadas por un movimiento obrero que surgía con fuerza en las zonas que se iban industrializando en España. Las huellas más lejanas de una actividad femenina dentro de la Prensa, según la profesora Asunción Bernárdez, se sitúan en el siglo XVII. La donostiarra Francisca de Aculodi fue la primera mujer en aportar su granito de arena al periodismo al publicar en San Sebastián entre 1687 y 1689 "Noticias principales y verdaderas", una revista quincenal que era una traducción al castellano de un periódico editado en Bruselas en lengua francesa por Pedro de Cleyn, a cuya traducción española se le añadían noticias locales y propias. Eran los comienzos de la prensa local. Francisca de Aculodi, da nombre desde 2014 a unos premios convocados por la Universidad del País Vasco. Se dice que fue la primera periodista de la historia. Así, esta mujer se adelantó por 19 años a la británica Elisabeth Mallet, fundadora en 1702 en Inglaterra Daily Courant, quien sin embargo es quien tiene el mérito internacional como primera mujer editora y periodista de la historia, rememora el periodista bilbaíno Itsaso Álvarez (Bernárdez Rodal 2015: 94).

En una sociedad jerárquica y clasista, como ha sido siempre la española, cuestiones como el sexo, el estado civil y la clase social determinan el lugar dentro de la sociedad. "si a esta óptica particular", denuncia la profesora de la Universidad de Almería Blasina Cantizano Márquez, "unimos un claro protagonismo masculino y la existencia de un sistema patriarcal que rige toda la sociedad, tenemos que la mujer ha sido considerada, hasta hace poco, como un ser secundario y débil que dependía de un hombretodopoderoso para poder participar dentro de la sociedad en la que vive y forma parte" (Cantizano Márquez 2004: 281-298). El recorrido por la Igualdad sigue siendo difícil y merece la pena plantearse las dificultades de aquellas pioneras con las de la sociedad de 2016. El concepto de inferioridad femenina, física e intelectual, fue una creencia generalizada, apoyada por un discurso religioso. 
“¡Las mujeres! ¿Cómo puede decirse que cuando las mujeres den señales de vida por la República se les concederá como premio el derecho a votar? ¿Es que no han luchado las mujeres por la República? (...) ¿Por qué el hombre, al advenimiento de la República, ha de tener sus derechos y han de ponerse en un lazareto los de la mujer?". Esa retahíla de preguntas de Clara Campoamor convenció a los diputados para aprobar el sufragio femenino. Ocurrió el 1 de octubre de 1931. Casi 100 años después, apenas hay huellas de la parlamentaria en la Cámara Baja. La sala bautizada con su nombre está en una entreplanta. Su busto aguarda en una parte del Congreso que no está abierta al público. Y su nombre no se menciona en las visitas guiadas, denunció el periodista Juan José Mateo, en enero de 2017.

La llamada Edad de Plata de la cultura española comprende desde el principio del siglo XX hasta el final de la guerra civil, cuando la represión y la censura acabaron con ese deslumbrante periodo denominado también "edad de oro del periodismo español", según el crítico Manuel Rodríguez Rivero. Una de las precursoras del feminismo, Carmen de Burgos Seguí "Colombine” (Rodalquilar, Almería. 1867-Madrid, 1932), hija del cónsul de Portugal en Almería, José Burgos Cañizares, se convierte en la primera mujer que se dedicó al ejercicio profesional del periodismo en España, mientras que en la radio esa figura la representa Josefina Carabias, como veremos más adelante. El primero director de El Heraldo de Madrid y, posteriormente, director de El Universal, Augusto Suárez de Figueroa (Estepona, 1852-Madrid, 1904), contrató como primera mujer redactora profesional de un periódico a la almeriense y a quien le cupo el honor de bautizarla como Colombine, según Manuel León (León 2016: 17). Colombine trabajó como redactora para el diario madrileño Diario Universidad en 1906 y cubrió episodios la Guerra de Marruecos (Rodríguez 2015: 1).

El año 1909 aún guarda otro acontecimiento decisivo para la vida de nuestra autora. Los hechos ocurridos en la Guerra de Marruecos, la masacre de soldados españoles en el barranco del Lobo, el 27 de julio de ese año, hace que en Barcelona se desencadene un alzamiento revolucionario en protesta contra la guerra, con la consiguiente represión por parte de las autoridades españolas. Miles de obreros levantaron las barricadas en las calles de Barcelona para evitar el reclutamiento forzoso decretado 
por el Gobierno. La situación en Marruecos se había deteriorado y el Ejecutivo había ordenado movilizar más tropas. Aunque todos los jóvenes estaban obligados a acudir al llamamiento, aquellos que pagaran un canon de 6.000 reales podían eludirlo. Una cantidad que solo las familias más pudientes podían abonar para evitar que sus hijos partieran al frente. Ante tal injusticia, la población se rebeló dando origen a la Semana Trágica. Lo que no sabían es que, a miles de kilómetros de allí, en las inhóspitas montañas del Rif, se estaba preparando una batalla que afectaría al devenir de los acontecimientos en la Ciudad Condal y que sólo interesaba a los propietarios de minas franceses y españoles asentados en el norte de Marruecos (ABC, 27 de julio de 2013.).

Carmen de Burgos, según la catedrática de la Universidad de Sevilla Carmen Ramírez, seduce por las muchas facetas artísticas y humanas que ofrece: "Mujer calidoscopio, siempre propone múltiples visiones del mundo y de la realidad de la mujer desde la plena conciencia de la verdad y de la libertad histórica e individual trasladando a su época, y aún a la nuestra, la plenitud de un pensamiento, de una sensibilidad y de una receptividad que no admiten silencios ni sombras. En ese sentido, su reflexión Daguerrotipo, 1931, en torno a la historia y a la fatal España inquisitiva empeñada en quemas de papeles y libros, traduce la lucidez de la preclara investigadora que sabe de una España que desatiende el progreso para permanecer estancada en la más absoluta inercia social, política y religiosa, agazapada en una secular ignorancia" (Ramírez Gómez 2000: 83-84), subraya la catedrática Ramírez quien se refiere a la escritora y periodista almeriense como mujer que contribuye a la construcción moderna de la identidad de la mujer contemporánea.

La actividad periodística en el Heraldo de Madrid se intensifica y Carmen no está dispuesta a dejar pasar esta nueva oportunidad. No duda ni un momento en trasladarse a Málaga y posteriormente a Melilla (Sevillano y Segura 2009: 37). Carmen se convirtió así en la primera mujer en aproximarse al conflicto, gracias a la credencial que le proporcionó Cruz Roja. En cambio, el Comisionado en el Norte de África prohíbía la presencia de mujeres en el campo de batalla. "Colombine en Melilla” será el titular directo e impactante que publicará el Heraldo de Madrid para dar cuenta de los sucesos más importantes, de la vida cotidiana de los batallones. 
Son decenas los autores que se han referido a la figura de Colombine. Se casó en 1833 con el periodista almeriense, Arturo Álvarez Bustos, hijo de un terrateniente. De naturaleza volcánica, como dijo Ramón Gómez de la Serna, quizá porque creció en un antiguo cráter de un volcán: el valle de Rodalquilar (Abad 2017). Su boda con el periodista Álvarez Bustos fue un episodio de ingrato recuerdo, comentó en una entrevista en La Esfera: "Lo motivó la equivocación más grande de mi vida. Mi rebeldía me llevó a casarme, contra la voluntad paterna". En un amplio reportaje la periodista almeriense Mar Abad, una de las confundidoras de la revista Yorokobu, trazó algunas claves de la difícil vida de Colombine en Almería. La escritora y profesora emérita del City College de Nueva York, Elizabeth Starcevic (Nueva York, 1942) fue la precursora en los estudios sobre Carmen de Burgos. Elaboró una tesis sobre la obra de la escritora almeriense y publicó en 1976 la biografía "Carmen de Burgos, defensora de la mujer", editada por Cajal, la innovadora editorial fundada por el añorado profesor José María Artero (Almería, 1921-1991), uno de los primeros que apostó por los temas andaluces desde Almería. Un periodista almeriense, Federico Utrera, es uno de los autores que rescató la obra periodística de Colombine: "Me pides que te traslade a Colombine al siglo XXI: creo que sería tan viajera como Soledad Puértolas, tan periodista como Maruja Torres y tan escritora como Almudena Grandes.

La Facultad de Ciencias de la Información de la Universidad Complutense le dedicó en 2010 un ciclo de conferencias. Lo importante es "el grupo de mujeres que en esos años intervinieron en el mundo del periodismo, la literatura y la política, y de las que no ha quedado ni rastro", afirmó Bernardino M. Hernando, archivero-bibliotecario de la Asociación de la Prensa de Madrid (APM). En este sentido, Hernando recordó a Jesusa Granda, otra almeriense, la primera mujer que ingresó en la Asociación de la Prensa de Madrid, con el número 67, a la también olvidada Atocha Osorio y Gallardo, la segunda periodista registrada en la APM, y a Consuelo Álvarez Pool, amiga de Carmen de Burgos, asociadas ambas con los números 1.029 y 1.030, respectivamente.

Sus intervenciones en la prensa nacional no tienen freno. Publica sus artículos en Diario Universal, Heraldo de Madrid, La Correspondencia de España, El País o revistas como Nuevo Mundo, La Esfera o Prometeo. Su trabajo literario lo centra en exponer las 
experiencias particulares que obtiene por el mundo en los sucesivos viajes que realiza por Europa. Así escribió Cartas sin destinatario y Peregrinaciones. Como poeta, llegó a influenciar a Federico García Lorca con su obra Puñal de Claveles, antecedente de Bodas de Sangre. Destacó por su lenguaje sencillo y la descripción de sus personajes (Torres Flores 2011: 19-26). Artículo femenino singular. Diez mujeres esenciales en la historia del articulismo español, la primera antología histórica de mujeres periodistas, dedica un capítulo a Colombine, considerada como una de las escritoras más sorprendentes, complejas y camaleónicas, viajera infatigable, feminista, pacifista y republicana hasta la médula. Todos estos adjetivos los expresó Helena Establier de la Universidad de Alicante, especialista en la obra de Colombine. La sede de la Asociación de la Prensa de Madrid reunió a varias autoras, en un acto celebrado el 13 de junio de 2011. La crónica de Sergio J. Valera recogió las palabras de la exdirectora de RTVE y actual responsable del Instituto Cervantes, la catedrática Carmen Caffarel: "No hay un periodismo femenino, sino una sensibilidad diferente para abordar algunos temas". Según Teodoro León Gross, codirector de la obra junto con María Angulo Egea, este proyecto se puso en marcha desde una doble perspectiva de género: el articulismo y la mujer. Ambos géneros coinciden en la exclusión y en la falta de conocimiento sobre ellos.

La profesora de la Universidad Rey Juan Carlos Concepción Núñez Rey es la más sólida biógrafa de Carmen de Burgos. Núñez considera a Mariano José de Larra como uno de los maestros de la periodista almeriense. "Visita su tumba y en 1919 publica en su honor Fígaro, una biografía que la consagra como una erudita. De esta manera alcanza su madurez y éxito académico. La escritora fue una adelantada de su época. Ya empezó a apostar firme por la necesidad de europeizar España. Asimila todo lo que lee acerca de Europa, que luego convertiría en crónicas excepcionales cuando viaja por todo el continente y nos traslada en sus textos las formas de vida de la sociedad europea y de sus mandatarios". La catedrática Núñez reiteró que la primera periodista profesional como redactora de Diario Universal (enero de 1903); corresponsal en Francia e Italia de Heraldo de Madrid y del naciente ABC (octubre 1905- verano 1906) ${ }^{3}$ En 1909, en Marruecos, se

\footnotetext{
${ }^{3}$ Homenaje a Carmen de Burgos en la entrega del V Premio Internacional Colombine, Almería, 28 de octubre de 2016.
} 
convirtió en la primera corresponsal de guerra y más tarde ofreció un extenso documento de la Primera Guerra Mundial. Destacó la profesora de la universidad Complutense que tras la muerte de Emilia Pardo Bazán, en mayo de 1921, Carmen de Burgos, se convirtió en "la decana de las letras españolas". La almeriense llegó a presidir la Cruzada de Mujeres Españolas y la Liga Internacional de Mujeres Ibéricas e Hispanoamericanas. Para la periodista catalana Teresa Soto Romero, ganadora del V Premio Internacional Colombine, el ejemplo de la periodista almeriense está en las profesionales que dan voz a los que no tienen y todas las que tienen compromiso solidario por mejorar las condiciones de vida de las mujeres ${ }^{4}$. Su reportaje ganador del premio El machismo que se ve, se emitió en La 2 de TVE en noviembre de 2015.

Colombine sigue acaparando la atención a lo largo del tiempo. Recogemos los últimos párrafos de un amplio artículo escrito en "infoLibre" por la periodista Saila Marcos: "Carmen de Burgos era una mujer muy conocida, escribía mucho en todos los periódicos y revistas, las mujeres la leían, tenía su público y se trataba realmente de una mujer importante. Además, tenía un salón literario en su casa del que habla Rafael Cansinos-Assens en La novela de un literato, integrado por tres o cuatro poetas de tercera fila y por Ramón Gómez de la Serna, su amante, que era gran apoyo para ella debido a su complicada vida personal, explica Anna Caballé, profesora de Literatura en la Universidad de Barcelona y experta en estudios biográficos. Pero en lo referente al reconocimiento literario por parte de sus colegas, era inexistente". Este es un texto que corresponde a un artículo publicado el 9 de enero de 2017 que concluye con el siguiente texto: "sobre Carmen de Burgos también han ido saliendo títulos a cuentagotas". A finales de los 2000 el Instituto de Estudios Almerienses reeditó Puñal de claveles (1931) o una breve novela sobre los mismos sucesos de Níjar que inspiraron a Lorca para escribir Bodas de sangre. En esa misma época aparecían dos biografías: la primera, de Blanca Bravo Cela, Carmen de Burgos. Contra el silencio (Espasa, 2003); y una segunda en 2005 firmada por Concepción Núñez Rey, profesora de Literatura de la Universidad Complutense. Asimismo, en 2012 Los libros de la Catarata reeditaba Mís viajes por

\footnotetext{
${ }^{4}$ Torres, Antonio. Noticia emitida por Canal Sur Televisión como homenaje a la periodista de TVE Teresa Soto, premiada por el prestigioso premio Colombine, emitido el 28 de octubre de 2016, 14.15 horas.
} 
Europa, la aventura de Colombine durante el verano de 1914 recorriendo desde Suiza a la zona más septentrional de Noruega" Caballé subraya que este proceso de recuperación no es un fenómeno nuevo: "Viene de atrás, quizás lo que ocurre es que en los últimos años se haya prodigado más", recoge Saila Marcos que concluye su artículo: "La reescritura del canon comenzó en la Transición, con artículos en revistas como Vindicación feminista, ensayos como La hora violeta, de Montserrat Roig, o los firmados por Carmen Martín Gaite. Además del trabajo fundamental de investigación que comenzaron a hacer feministas como Shirley Mangini o Susan Kirkpatrick en las universidades estadounidenses (antes, incluso, que en las españolas). Todo esto surge por una cuestión de necesidad. Si hablamos de las escritoras españolas, necesitamos saber de dónde vienen, de manera que ha habido que reconstruir una fotografía que no estaba en ninguna parte y colocarla en la historiografía literaria. A pesar de las sucesivas olas de recuperación, el mayor inconveniente, señala Caballé, es el legado personal de estas mujeres, perdido en muchos de los casos. ¡Lo que daríamos ahora por leer las cartas de Carmen de Burbos!, se lamenta. Mientras tanto, uno de los mejores acercamientos a la intimidad de Colombine lo podemos encontrar en La malcasada, donde conviven su mundo de ficción con la angustia de las frustraciones y anhelos que determinaron su vida personal y profesional" (Marcos 2017.).

\section{Josefina Carabias y Julia Calleja entran en La Palabra}

La radio nació como un altavoz musical para extender al gran público las audiciones de conciertos, óperas y música popular. El diario hablado La Palabra se creó en Unión Radio, en 1930, como el primer informativo de la radio. Josefina Carabias (Arenas de San Pedro, Ávila, 1908-Madrid, 1980) entró a trabajar como redactora de ese primer informativo en 1932. Carabias es la primera mujer española que tuvo como tarea principal el periodismo, a diferencia de sus antecesoras, que eran escritoras o colaboradoras (Torres Flores 2011: 27-31). Carabias tenía una relación laboral de plantilla en Unión Radio Madrid. Carabias acudía diariamente a la redacción en los años de la República. Su trabajo fue incansable hasta el momento de su desaparición. En los años 
cincuenta se convirtió en una de las primeras corresponsales en el extranjero y acabó su carrera profesional siendo una columnista de lujo. Es la primera redactora de programas informativos de la radio. Era a los 20 años pionera del periodismo escrito y radiofónico, tras abandonar los latifundios de sus padres, para estudiar Derecho en Madrid. Se instala en la Residencia Internacional de señoritas que llevaba el nombre de María de Maeztu (Vitoria, 1882-Buenos Aires, 1948). La Residencia de Señoritas, creada en 1915, potenció el progreso de la mujer. La institución formó a una generación vanguardista, escribió Tereixa Constenla (2015: 31). La Residencia tenía la meta de proporcionar una casa y un lugar de estudio a las jóvenes de provincias. Para Carmen G. de la Cueva, periodista del semanal Ahora, dirigido por Miguel Ángel Aguilar y que cerró sus puertas en octubre de 2016, "Pardo Bazán fue una precursora de las modernas de Madrid y denunció la situación de la mujer" (G. de la Cueva 2016: 18-19.). En cambio, en pueblos del Sur no había ni escuelas.

Carabias se codea con personajes de su tiempo, como Azaña, Azorín, Baroja, Indalecio Prieto o con el economista y abogado José Rico Godoy, quien años después sería su marido. Rico Godoy era almeriense, al igual que una de las precursoras del feminismo en España, Carmen de Burgos "Colombine", primera redactora en nómina de un periódico y corresponsal de guerra en África. La primera redactora de la radio en nómina fue la referida Josefina Carabias. Carabias escribe su primer artículo en 1931, en la revista madrileña Estampa, dirigida por un primo suyo, y pronto entraría a formar parte de la redacción. Ese año inicia su colaboración con el diario Ahora, también de Madrid. En 1932, aprueba las oposiciones para registradores de la propiedad y, al año siguiente, entra como cronista parlamentaria en el periódico La Voz. A partir de este momento, vivirá de cerca debates parlamentarios en los que también participaban las mujeres como diputadas. En 1933, se incorpora a la redacción de La Palabra, diario hablado matinal de Unión Radio. Se convierte en la primera locutora de noticias de España, la primera redactora dentro de un informativo propiamente dicho como La Palabra. Su primer gran éxito como periodista fue la publicación, en 1931, de una entrevista que había realizado a la jurista malagueña Victoria Kent Siano (1889-1987), que por aquel entonces estrenaba su flamante nombramiento como Directora General de Instituciones Penitenciarias. Esta 
entrevista, muy leída y comentada en los medios políticos y culturales del país, impulsó definitivamente la carrera periodística de Josefina Carabias: comenzó a alternar sus colaboraciones en La Estampa con otros artículos aparecidos en el rotativo La Voz y en los semanarios Crónica y Mundo Gráfico, medios cuya importante difusión contribuyó a hacer de Josefina Carabias una de las periodistas más populares del período republicano.

A su regreso a España, se encontró con un enorme vacío a su alrededor, sólo quebrantado por la acogida que, entres sus páginas, le ofreció el diario Informaciones. Pero sus conocidas simpatías políticas impidieron que su afilada pluma abordara durante aquellos difíciles años de la posguerra los mismos temas que había tratado durante la Segunda República, por lo que la dirección del rotativo tuvo la afortunada ocurrencia de proponer a Josefina Carabias que escribiera crónicas futbolísticas. Aquí Carabias, se convierte también en una de las mujeres pioneras en el tratamiento del fútbol como espectáculo en los medios de comunicación. Se reveló, así, la escritora abulense como una periodista de raza, capaz de salir adelante en cualquier sección de un diario: sus crónicas sobre la Liga de Fútbol tuvieron tal aceptación, que al poco tiempo fue necesario agruparlas en el volumen titulado La mujer en el fútbol. Gracias a esta acertada iniciativa del rotativo Informaciones, Josefina Carabias volvió a recuperar parte del predicamento de que había gozado en los principales medios de comunicación de épocas anteriores. Su "rehabilitación" definitiva -a ojos de las autoridades del nuevo régimen político-, vino en 1952 con la concesión del premio periodístico "Luca de Tena", al que siguió, al cabo de dos años, el no menos reconocido "Mariano de Cavia". Carabias y otras pioneras españolas son las protagonistas del documental Nosotras que contamos, dirigido por Inés García-Albi, emitido en La 2 de TVE (Torres Flores 2016: 129-149).

La forma de recuperar a Josefina Carabias para la información política y social consistió en enviarla a Washington como corresponsal del diario Informaciones en la capital norteamericana, donde halló ocasión de retomar los temas de sus antiguas crónicas sin incidir directamente en la delicada situación interna del país. A su regreso a España pasó a formar parte de la redacción del diario católico Ya, cuya dirección, decidida a sacar partido de su antigua estancia en Francia, pronto la puso al frente de su corresponsalía en París. Desde 1959 hasta 1967 permaneció en la capital francesa Josefina Carabias, donde 
escribió gran cantidad de crónicas y reportajes que, muy difundidos por toda España, volvieron a convertirla en una de las voces femeninas más descollantes del periodismo contemporáneo. Fue la periodista que cubrió para los periódicos de la Editorial Católica, Ideal o La Verdad de Murcia, la primera boda mediática de la Fabiola y Balduino. En la memoria llevaba que la guerra civil frustró muchas carreras y no solo en el ámbito de la comunicación y el periodismo.

La Voz será un vespertino popular que, no siendo sensacionalista y estando bien escrito, ocupó el primer puesto en la venta callejera, alcanzando en 1930 los 130.000 ejemplares, casi la mitad de ellos en provincias, con notas editoriales breves y ligeras y dedicando gran atención a los sucesos o a los toros y una sección diaria dedicada al movimiento obrero. Muy ilustrado, su viñeta diaria en primera se debía a la pluma de Manuel Tovar y Alfonso fue uno de sus principales fotógrafos. Siendo un diario de información política y de noticias nacionales, de provincias y extranjeras, publicó también el tradicional folletín, y obtuvo importantes ganancias por los anuncios y la publicidad $^{5}$. Estuvo dirigido por el periodista granadino Enrique Fajardo (Granada, 1883México, 1948), conocido como Fabián Vidal, y su redactor jefe será Manuel Bueno, que será reemplazado por Benito Artigas Arpón en 1931, cuando el diario cambie de manos empresariales. La Voz tuvo que suplir en algunos momentos a $\mathrm{El} \mathrm{Sol}$, que pertenecía a la misma empresa, cuando este fue suspendido, editando incluso ediciones matutinas, y en 1937 incluirá los jueves El mono azul. Los talleres de La Voz, que compartía con El Sol, fueron confiscados en 1939 por Falange para editar su periódico falangista Arriba. A la novelista y periodista de El País, Rosa Montero, Isabel Gómez de la revista Jot Down, le preguntó qué modelos femeninos le ayudaron a construir tu identidad profesional como periodista: "Modelos femeninos", confesó Montero:

había realmente poquísimos. Estaba Josefina Carabias, estaba... pues... [piensa] Pues Josefina Carabias [risas]. Ah, bueno, también leí, por ejemplo, ya con 17 años, a Nativel Preciado. Ella comenzó dos años antes que yo, en el Madrid, y pensaba "mira qué bien ésta". Poco después nos hicimos amigas. Así que somos amigas desde hace 40 años, la adoro. Nativel, Carabias y también la fotógrafa Christine Spengler, que ha sido sobre todo fotógrafa de guerra, pero que entonces, cuando yo tenía 17 o 18 años, también estaba en no sé qué guerra escribiendo unas crónicas

\footnotetext{
${ }^{5}$ Biblioteca Nacional de España. Hemeroteca digital.
} 
que leía y decía aaaah... Y poco más. Después, cuando había empezado a escribir, cuando ya era periodista, leí por supuesto a Oriana Fallaci. ¿Modelos? No eran exactamente modelos. Yo escribía desde muy pequeña. La mayoría de los novelistas empezamos de niños: la escritura forma parte de la estructura básica de la personalidad. Desde los cinco años escribía, así que, de alguna manera, aunque recuerdo a estas periodistas... Yo decía: se puede ser, ¡claro, estaban escribiendo!, se puede ser... ¡Ah! Otra que fue un modelo para mí: Soledad Alameda, un poco mayor que yo, que hacía entrevistas en Fotogramas. (Gómez Rivas y Rosa Montero 2013)

Por su parte, otro referente del periodismo como Maruja Torres, tuvo en sus inicios las lecturas de Carabias desde El Noticiero Universal, extinto periódico que se editaba en Barcelona: "Las hacía desde Washington y París”, citó Torres, "y a Martín Girard, que resultó ser Gonzalo Suárez, con sus estupendas crónicas de vestuario de fútbol. Leía y sin saberlo estaba buscando un estilo, buscaba hacer las cosas de una forma personal, implicándome y con un punto de vista diferente" (Bernal 2012).

Colombine y Carabias fueron referentes para las que vinieron después como Pilar Narvión, la primera en ejercer crónica política dentro de la España en blanco y negro. A finales de 2016, el mérito es evidente con porcentajes de mujeres con estudios universitarios del $43 \%$ frente al $36 \%$ entre los hombres. La brecha salarial y el techo de cristal siguen teniendo retos para las mujeres en España. En el panorama de 2017 es necesario contar con referentes. Los procesos de cambio social necesitan de un nuevo comunicador que facilite el diálogo intercultural. El perfil de este comunicador es una suma de conocimientos y experiencias que, por el momento, no se ofrecen en los programas de las universidades. La necesidad del nuevo comunicador existe. La demanda crece en las organizaciones de desarrollo y en las propias comunidades, pero no hay una oferta clara, vaticina Alfonso Gumucio, director ejecutivo del consorcio de comunicación para el cambio social y especialista en comunicación para el desarrollo con experiencia en África, Asia, América Latina y el Caribe.

Se pueden contar en 2017 el número de directoras de medios de comunicación. Hubo una oportunidad histórica para que el liderazgo de El País lo encabezara una mujer periodista y muy prestigiosa. Soledad Gallego-Díaz (Madrid, 1951), quien fue adjunta 
con los distintos directores Juan Luis Cebrián, Joaquín Estefanía y Jesús Ceberio, pudo llegar a ser la primera directora de El País. Declinó la oferta. El actual consejero delegado de Prisa, editora de El País, Juan Luis Cebrián (Madrid, 1944), en su libro de memorias, editado en 2016, lo certifica. Cebrián fue el primer director del diario y quería dejar en el cargo a Soledad Gallego-Díaz como su relevo al frente del periódico:

No tuve dudas de proponer a mi directora adjunta, Soledad Gallego-Díaz. Sus primeros pinitos como reportera los había hecho en Pyresa, la agencia de prensa del Movimiento, bajo la dirección de mi padre. A la hora de conformar la redacción del periódico él me aseguró que no me equivocaría si la contrataba a ella y a Bonifacio de la Cuadra, también antiguo colaborador suyo. Seguí el consejo al pie de la letra y los resultados no pudieron ser más satisfactorios. La personalidad de Sol, su dedicación al trabajo, su extensa cultura, su entusiasmo y su candor, no exento de dureza, también su popularidad entre los profesionales, me parecían condiciones de ensueño a la hora de sustituirme y mejorar lo que consideraba mi obra. Con gran decepción por mi parte rechazó un ofrecimiento para el que no tenía alternativa clara, pues el otro potencial sustituto, Antonio Franco que había dirigido la edición catalana desde su lanzamiento en 1982, nos acababa de abandonar para retornar al frente de El Periódico de Catalunya; y no podía prescindir del papel de Delkáder en la SER, ahora que me iba a hacer cargo de ella, habida cuenta de las desconfianzas que padecíamos respecto a su consejero delegado. Decidimos iniciar un debate con los subdirectores y redactores jefes, todos ellos candidatos posibles al puesto dada su excelente trayectoria, aunque no fuéramos capaces de pronunciarnos por ninguno en particular. Del diálogo pasamos a una reñida votación. El mejor considerado, por un margen relativamente estrecho, fue Joaquín Estefanía, que se haría cargo del periódico durante los cinco años posteriores. Desde aquel día, hemos conseguido la norma invariable de nombrar a los directores de El País (cinco hasta ahora, contando conmigo) entre el equipo de la casa. Nos han acusado de que semejante práctica acarrea cierta endogamia perjudicial para el periódico mismo, pero son tan fuertes la identidad de la marca y la coherencia de su evolución durante más de cuarenta años de vida que la irrupción de un paracaidista, por hábil que fuera en el aterrizaje, nos ha parecido siempre rechazable". (Cebrián 2016: 378)

Unos años antes del citado libro de Cebrián, la periodista Gallego Diez recordó:

Precisamente por haber decidido no aceptarlo no creo que lo deba comentar. Tampoco creo que hubiese sido un periódico muy diferente de haberme hecho cargo yo de él. Desde el despacho de adjunta mandas un huevo". Lo que no puede negar es que hubo un momento en el que quienes llevaban las riendas se dieron cuenta de que cada vez debían dar más cabida a las mujeres". ${ }^{6}$

\footnotetext{
${ }^{6}$ www.JotDown,.es, 23 de marzo de 2012.
} 
De los 221 periódicos que hay en España, solamente 20 son dirigidos por mujeres y de los 15 más importantes de papel, sólo uno, La Nueva España tiene una directora, dijo la decana de la Facultad de Ciencias de la Información, Carmen Pérez de Armiñán. Durante el encuentro Periodismo por Mujeres, organizado por estudiantes de cuarto curso de Periodismo miembros del programa La Cueva de Inforadio, en colaboración con el Instituto de Investigaciones Feministas de la UCM, la asignatura de Género de la Facultad y el colectivo Letras Feministas, Pérez de Armiñán, agregó que eso es así a pesar de que los estudios de comunicación cada vez cuentan con más mujeres matriculadas ${ }^{7}$. De acuerdo con datos de la decana, hoy en día más del 60 por ciento de la matrícula son mujeres, un crecimiento exponencial desde la primera promoción del año 1971 donde se ubicó en $28 \%$.

\section{Techo de hormigón}

Para encontrar a una mujer dirigiendo un periódico impreso en España hay que descender hasta el número 23 de la lista de medios más leídos del Estudio General de Medios. Se trata del diario económico Expansión, dirigido por Ana I. Pereda, y al 24, posición que ocupa El Día, de Tenerife, cuya dirección y edición recae en María Mercedes Rodríguez., desde abril de 2014. El accionariado del periódico está en manos de tres personas, la propia Mercedes Rodríguez (33\%), su hija Adriana de Lorenzo Cáceres Rodríguez y el hermano de Mercedes José Esteban Rodríguez. La dirección y la edición del periódico fueron encomendadas a Mercedes Rodríguez, por expresa voluntad de su padre, que ya en vida la había nombrado editora adjunta, según la información de El Diario de Tenerife (14 de abril de 2014). Por otro lado, se encuentran el diario Granada Hoy con Magdalena Trillo (Torres Flores 2011), La Nueva España de Oviedo con Ángeles Rivero Velasco, periodista con la que me entrevisté en julio de 2014, en su despacho de la redacción central ovetense. También es digno destacar a Esther Vera (Badalona, 1967), al frente de Ara. La ausencia de mujeres en los puestos de dirección sorprende, sobre todo, cuando se compara con la cifra de trabajadoras en los medios. El

\footnotetext{
${ }^{7}$ Cimacnoticias/AmecoPress, 18 de enero de 2017.
} 
51"8\% de todos los periodistas con contrato de trabajo pertenecen al género femenino. En el caso de los profesionales autónomos o free lancers el total de mujeres es del 40,3\%. Hay una evidente desigualdad. “QQué pasa? Que tenemos unos patrones muy masculinos en la prensa española que condicionan y que influyen, por ejemplo, en que la mayoría de los directivos sean hombres y que éstos elijan a otros varones para dirigir los medios continuando la dinámica”, señaló a Público Malén Aznárez (Santander, 1943, presidenta de Reporteros Sin Fronteras-España, primera mujer en dirigir una cadena de periódicos en este país y también primera directora de los Servicios Informativos de RNE a mediados de los años ochenta. En el periodismo digital, la presencia de mujeres va en aumento. En La Marea, medio que dirige Magda Bandera, los cuatro puestos de mayor responsabilidad son mujeres. O en Público, donde la adjunta a la dirección, Virginia P. Alonso, es también mujer. "No hay mujeres al frente de los medios porque no hay mujeres al frente del poder", señaló Ana Pardo de Vera. Para las directoras, la ausencia de mujeres en los puestos directivos de los medios no es más que el reflejo de la sociedad donde el poder económico y político sigue estando profundamente masculinizado. Para la actual directora de la editorial Hotel Papel, Nuria Varela (2017), el concepto de techo de cristal es un eufemismo: "Es un techo de hormigón”.

\section{Referencias bibliográficas}

Abad, Mar. 2017. "Carmen de Burgos, la escritora y activista que Franco borró de la historia". Yorokobu. http://www.yorokobu.es/carmen-de-burgos/ [30 mayo 2017].

Bernal, Jordi. 2012. "El periodismo está propiciando el miedo de la sociedad”, Jot Down, abril, 2012.

Bernárdez Rodal, Asunción. 2015. Mujeres en medios. Madrid: Editorial Fundamentos.

Cantizano Márquez, Blasina. 2004. "La mujer en la prensa femenina del siglo XIX". Ámbitos, $\mathrm{n}^{\circ}$ 11-12 (2004).

Cebrián, Juan Luis. 2016. "Primera página. Vida de un periodista 1944-1988”, Debate (2016): 378.

Constenla, Tereixa. 2015. "La casa de las habitaciones propias". El País, 2 de diciembre de 2015: 31 . 
G. de la Cueva, Carmen. 2016. "Modernas, las mujeres que no se quedaron en casa". Periódico semanal Ahora, 8 de enero de 2016: 18-19.

Gómez Rivas, Isabel y Rosa Montero. 2013. "Las redacciones han terminado convirtiendo en fortines apaches", Jot Down, febrero de 2013.

León, Manuel. 2016. “Los que se batían en duelo". La Voz de Almería, 20 de noviembre de 2016: 17.

Marcos, Saila. 2017. "El turno de Carmen de Burgos". infoLibre, 9 de enero de 2017.

Ramírez Gómez, Carmen. 2000. Mujeres escritoras en la prensa andaluza del siglo XX. Sevilla: Universidad de Sevilla.

Rodríguez, Marta. 2015. “Carmen de Burgos, entre las más influyentes del siglo XX”. La Voz de Almería, 21 de abril de 2015: 1.

Sevillano, Antonio y Segura, Anyes. 2009. Carmen de Burgos "Colombine” (Almería, 1867-Madrid, 1932). Almería: Instituto de Estudios Almerienses.

Torres Flores, Antonio. 2011. Mujeres de palabra. Crónica de las pioneras en femenino. Prólogo de Pilar del Río. Almería: Instituto de Estudios Almerienses.

Torres Flores, Antonio. 2016. "Mujeres invisibilizadas en las redacciones: El escaso reconocimiento profesional de las profesionales del periodismo en España". En Cristina Prieto (ed.) Periodistas ante el Derecho a la Comunicación de la Ciudadanía. Sevilla: Egregius Ediciones y Facultad de Comunicación.

Varela, Nuria. 2017. "Es un techo de hormigón”. Cimacnoticias/AmecoPress, 18 de enero de 2017. 